\title{
The thinking on "Zhi" in Zhuangzi
}

\author{
Rongkun Zhang \\ The Hong Kong University of Science and Technology \\ Hong Kong, China
}

\begin{abstract}
Zhuangzi is a philosopher who consciously ponders on "Zhi" in the Pre-Qin Dynasty. It constitutes an important part of Zhuangzi's philosophy and becomes a significant way to understand Zhuangzi. This essay is based on two propositions- "Zhi Wei Nie" and "Yi Zhi Wei Shi" to discuss "Zhi". As far as Zhuangzi is concerned, "Zhi" is an instinctive ability but we cannot always conform to "Tian", so "Zhi" becomes a "weapon" that we use to struggle with each other due to our selfish motives. This is the meaning of "Zhi Wei Nie". On the contrary, "Yi Zhi Wei Shi" is a right living way which eliminates egoism, utilitarianism and instrumentality. As a consequence, "Zhi" is a basis on which human is in harmony with the heaven (tian) and deserves the title "Tian yu".
\end{abstract}

\section{Keywords-Zhuangzi; zhi; heaven (tian); way of living}

\section{INTRODUCTION}

"Zhi" is the inherent potential of people, and it is also an important sign of people distinctive from other things, so it has great significance for "people". Zhuangzi is a philosopher who has a rather conscious and quite complete thinking about the "Zhi" of human in the pre-Qin period. The word "Zhi" appeared in the "Zhuangzi" for about 157 times, and in many cases Zhuangzi had a clear philosophical consciousness for use of this vocabulary. He found that "Zhi" as people's inherent potential had a positive part, but also had negative part; meanwhile, Zhuangzi perceived that "Zhi" not only contained the personal significance for individual person, but also had the significance in social ethics and politics, and even the significance of coexistence between people and all other things on the universe. In the view of Zhuangzi's philosophical consciousness of "Zhi", "Zhi" theory actually constitutes an important part of Zhuangzi's thought, and also emphatically embodies the inner tension of Zhuangzi's thought, which is an important way to understand Zhuangzi's thought. Therefore, the further discussion and study on Zhuangzi's thought in "Zhi", is not only beneficial to our understanding of the intrinsic "Zhi" for human, but also good for our comprehension and grasp of Zhuangzi's thought, so it has important philosophical significance.

On the "Zhi", "Zhuangzi · Waiwu" cited Zhuangzi’s words:

A man having sharp foresight is entitled wise man, having sensitive hearing called acute man, having sensitive nose called frank man, having sensitive taste called willingly man, having clear mind called intelligent man, having clever comprehension chaste man. In general, a man with morality does not want to be congested, because congestion will cause obstruction, and when obstruction can not be solved, there will be trampling each other, and then a variety of scourge will follow. Perception depends on atmosphere, and if the atmosphere is not strong, it is absolutely not resulted from natural endowment. The truth of nature runs through all things, continuously going days and nights, but people may block their own opening of the body. There are many empty areas in the abdomen so fetus can be held among the five internal organs; if a man has weak mind, he will drift along in compliance with the nature. If there is no vacancy in a house, there will be quarrel between the mother-in-law and daughter-in-law; if a man has great mind but is not selfcritical for himself, his six senses will be troubled. ${ }^{1}$

In Cheng Xuanying's Theory on Dredge, it is said: thoroughness means smoothness ${ }^{2}$. In Zhuangzi's viewpoint, as a man with smooth eyes, ears, nose and mouth can clearly see, hear, smell and taste, "Zhi" is a capability after "mind" gets smooth. And in the words "if a man has great mind but is not self-critical for himself, his six senses will be troubled", the proper operation of "mind" directly decides the proper operation of eyes, ears, nose and mouth (six sense organs). By this, as the power of "mind", "Zhi" is a capability higher than the function of seeing, hearing, smell and taste (of six sense organs) but also can dominate them. Thus, as the capability of "mind", "Zhi" actually constitutes the foundation of a person to deal with things. However, in the view of Zhuangzi, the "Zhi" from "mind" must be treated from both sides, and such as in " a man having clever comprehension chaste man ", if "Zhi" is thorough and smooth, all things will be done properly and orderly; whereas, if "people may block their own opening of the body" and "a man has great mind but is not self-critical for himself", the "Zhi" will be incapable and causes "six senses will be troubled", and people can not deal with things orderly and properly. For the above-mentioned positive and negative aspects, Zhuangzi had a clear distinction and repeated discussion in the "Chapter of Inner", for which it is generalized that Zhuangzi called the former "Zhi is opportunity"3 but denounced the latter "Zhi is evil". ${ }^{4}$ This

\footnotetext{
1 Guo Qingfan: "Zhuangzi Ji Shi" (Beijing: Zhonghua Book Company, 2004), p939. This paragraph is out of miscellaneous articles, but there is the words of "Zhuangzi Says" and the thought discussed is very consistent with that in the inner chapter, so we think it can be used as supplementary document of the inner chapter.

2 Guo Qingfan: "Zhuangzi Ji Shi", p939

3 Guo Qingfan: "Zhuangzi Ji Shi", p234

4 Guo Qingfan: "Zhuangzi Ji Shi", p217
} 
article is purposed to make a further discussion on Zhuangzi's distinction and thinking on "Zhi" based on the positive and negative propositions given by Zhuangzi.

\section{II. “ZHI IS EVIL" FOR COMMON PEOPLE}

Before analyzing the positive discussion of Zhuangzi on "Zhi”, we will first explain and analyze Zhuangzi's criticism and reflection on the misuse of "Zhi" among the common people, because only after making a deep understanding and thinking about the misuse of "Zhi" among the people, can we clear out all the obstacles in our idea and then have a tangible understanding of the "Zhi" in the positive discussion by Zhuangzi.

Zhuangzi has a lot of criticism in the chapter of inner, we think the saying of "Zhi is evil" is a concise and precise conclusion of Zhuangzi in all kinds of criticism, so we take this proposition as a breakthrough point to understand Zhuangzi 's criticism of "Zhi”.

The saying of "Zhi is evil" comes from a paragraph of discussion under two fables in "De Chong Fu".

In the paragraph of discussion, Zhuangzi explained that people should develop their virtue and forget the shape first through the two fables of "Yin Qi Zhili Wu Shen Shuo Wei Ling Gong" and "Weng Ang Da Ying Shuo Qi Huangong", and then distinguish the "De" and "Xing" by means of "Tian" and "Ren": if "heaven" is taken as the nature of things, without artificial behavior, the "De" can be called "heaven virtue"; if "Ren" is taken as various ardour and dislikes of things due to expediency, various artificial behaviors will be caused. In this section, "no feeling of human" is used to describe the idea of "Ren" entering into the "Tian". In the discussion between Zhuangzi and Hui Shi about "people have no feeling" at the end of the paragraph, Zhuangzi made a more specific explanation: "no feeling" should come from the nature, people should not produce affection, dislike and artificial behaviors due to expediency, or else it will "injure their body" . Just based on this, Zhuangzi criticized the imposture and misuse of "Zhi", "Yue", "De ${ }^{6 "}$ and "Gong" due to people's expediency and dislike. From the viewpoint of Zhuanzi, the "Zhi", "Yue", "De" and "Gong" before misused are "Tian yu", the endowment of the nature for people to live in the world, but people ${ }^{7}$ impose it to realize their expediency and like. Here, the endowment of the nature also has a certain content of tools and means, by virtue of the meaning of "nature", but it is limited by its "nature", so the saints can use the "Zhi", "Yue", "De" and "Gong" according to the rules of the nature, and not take it as a special means to realize their private purpose. The saints can follow the rules of the nature and won't disturb and destroy it, so the saints won't destroy its "nature" due to their purpose. More precisely, the purpose of the saints is inherently an organic part of the natural process of all things in the world. on the

\footnotetext{
5 Guo Qingfan: "Zhuangzi Ji Shi ", p221

6 The "De" here has already been used by people for their selfishness, different from the "Tian De" of the sage.

7 "Zhuangzi often take "common people" as the opposite to the "Zhenren", See Guo Qingfan: “Zhuangzi’s Ji Shi”, p11,83,100,228,268.
}

contrary, common people take the "Zhi", "Yue", "De" and "Gong" as tools to realize their expediency so as to make them only have the value of tool to realize individual expediency. Just as discussed by Zhuangzi, people consider and use "Zhi" for their expediency, so they lose the harmony with the nature and impose the "Yue" in breach of the rule of the nature, then the "De" and further the "Gong" like a peddler for their individual interest (Huo).

Just for the above-mentioned insight that "Zhi" is used as a tool for people to make artificial behaviors and the endowment of the nature is change, Zhuangzi gave out his sigh of "Zhi is evil" to describe the "Zhi" for common people. It mainly has two kinds of interpretations, of which one is to "evildoer", such as the saying "wisdom producing evildoer" of Sima Biao cited in Shi Wen ${ }^{8}$, and the other is "offshoot", such as Lyu Huiqing's saying "if taking Zhi as evildoer, evildoer is produced from trunk rather than being the trunk", and Lu Shuzhi's saying "if taking wisdom as evildoer, the evil branch of wood or the evil son of human is not authentic"10 , and Zhong Taiyun's saying "for the persons of 'Zhi is Evil', Zhi is unwanted, like the redundant tree branch, not the trunk" ${ }^{\prime 1}$, both expressing this meaning. In fact, the above explanation is both two-into-one and one-into-two. People take "Zhi" as a tool to realize expediency, not following the rule of the nature "Tian yu", so it is offshoot, superabundant and not prime. Common people's "Zhi" becomes superabundant, so it will obstruct and injure the nature of people and the nature of things, and further the adverse consequences produces and "evildoer produces".

In fact, Taking "Zhi" as a tool and means to realize expediency is using "Zhi" and "Mou" to strive for what you want, so Zhuangzi gave the saying of "Zhi comes form fight" in his Renjian Shi.

Do you know how the moral is destructed and the wisdom is shown? The destruction of morality lies in the pursuit of fame, the display of wisdom is to argue right and wrong. The fame is the reason of mutual strife, and wisdom is a tool of struggle with each other. Like lethal weapon, neither of them should be carried in the word. ${ }^{12}$

Here, the "The destruction of morality lies in the pursuit of fame, the display of wisdom is to argue right and wrong", similar with the "morality is for connection and Zhi is evil" in De Chong Fu explains the "De" and "Zhi" in exterior, utilitarian and implemental state by common people.

For the above "Zhi" and "Mou" in utilitarian and implemental state as well as the "'Zheng" associated with them, Zhuangzi had a very particular description with deep hatred and resentment in the Essay on the Uniformity of All Things.

\footnotetext{
8 Guo Qingfan: "Zhuangzi Ji Shi", p218

${ }^{9}$ Lyu Huiqing: "Zhuangzi Yi Ji Xiao" (Beijing: Zhonghua Book Company, 2009), p.109.

${ }^{10}$ Lu Shuzhi: "Zhuangzi Xue" (Shanghai: East China Normal University Press, 2011), p.67.

11 Zhong Tai: "Zhuangzi Fa Wei" (Shanghai: Shanghai Ancient Books Publishing House, 1988), p.124.

12 Guo Qingfan: "Zhuangzi Ji Shi ", p135
} 
A talented person is open-minded, and only the persons with petty trick are willing to haggle over every ounce. The speech catering for the public is full of arrogance, and the saying sticking to giff-gaff is trivial and endless. When sleeping, they are disturbed in mind, and after waking up, they are uneasy in body, tangled with others, infighting every day. Some of them are hesitant and slow, some are unfathomable, and some are cautious. They are anxious and fearful or terrified and lose their mind. Their words are swift and acerbic as if the arrows leave the crossbow, from which quarrel are brought. If they hide thinking in their mind as if they abide by their covenant and vow, they are waiting for the opportunity of victory. If they get decay like the grass in autumn and winter, they are increasingly destroyed; if they indulge in all the things they engaged in, they may not be restored to the original situation; if their minds are bounded, they are declining and can not make them back to vigor. They are happy, angry, sad, joyful or they worry, sigh, repeat, fear; they are restless frivolous, luxurious, indulgent, insolent, deceitful. As if the music is gave from the hollow music tube but also as if is formed by bacteria through telluric effluvium transpiration. These kinds of modality appear and are replaced each other all days and nights, but no one know how it produces Forget it, forget it! Once you understand the truth of all these, you will know the reason that these modalities appear and form?...13

Once forming the body according to the rule of the nature, they will not forget their selfinterest and wait for the final death. They oppose to the world or comply with the world, they act as a rapidly racing horse, and nothing can stop them. This is very sad! They are always in service but do not see their success; they are tired in life but they do not know their own end. This is sad! People say that such persons can not die, but what benefit is for this! Human body is gradually depleted, the spirit and feelings of people is also crocking up together. This is the greatest sorrow, isn't it? a man, living in the world, is ignorant originally like this? Is it only me to be so ignorant? Others are ignorant too! ?

This word is continued after the "Tian Lai" "Di Lai" "Ren Lai"14 of Essay on the Uniformity of All Things. In the fable, Zhuangzi cited the dialogue between Nanguo Ziqi and Yancheng Ziyou to state that all the sound from the grotto in the mountain forest is affected by itself and it is originally produced by empty. By this, Zhangzi made metaphor of "Zhi" and "Mou" of people due to their expediency and various fakes resulted by the "Zhi" and "Mou", and described various states of people in life such as awaking and sleeping, connection and association, fear and dread, stop and so on, and gave sadness and sorrow for these. In the summary of this kind of emotional state, Zhuangzi used the words of "oppose to or comply with things" to profoundly point out that "Zhi" vainly became the "weapon for fight" after being taken as a means like tool, while he used "do not see success" and "do not know their end" to point out that people had no dependence and life passion and further became vain after they are narrow-minded for self-interests.

\footnotetext{
13 Guo Qingfan: "Zhuangzi Ji Shi ", p51, 56

14 Guo Qingfan: "Zhuangzi Ji Shi ", p43-50
}

In contrast, the ideal life modality in the mind of Zhuangzi is the "ancient Zhengren", "who has delight and wrath are natural like the change of four seasons, in compliance with the things in the world, and no one can know his spiritual truth" and "who does not dream when sleeping; not worry when waking up; not ask for cate when eating; and runs deep when breathing." In the view of Zhuangzi, this is called the "secret of nature" is got, while the reason why the people lost their "secret of nature" is "they blindly indulge in sensory enjoyment so as to have shallow wisdom" 15 . Here "indulge in sensory enjoyment" means strong expediency and it makes the natural "Zhi" that is originally the resource of nature ("Tian Yu") change into a tool and means of people to realize their expediency. Just for his profound view of such life modality as "blindly indulge in sensory enjoyment for expediency", Zhuangzi often uses the language beyond extraordinary people's imagination to describe the idea life modality that is opposite to this.

In the distant Gushe mountain, lived a man of God, having smooth and white skin like snow and ice and mellow posture like virgin, who does not eat the grain but drink refreshing breeze and nectar ... for such a man, nothing is able to hurt him, the monstrous flood can not drown him; the serious drought can make the stone melt and the earth burnt but he did not feel hot. ${ }^{16}$

As a man of accomplishment, once you get the truth, are simply immortal. When the world is on fire, you will not feel hot; when the river is frozen into the ice, you will not feel cold; when there are thunder, tornadoes and tsunami, you don't fear. Such a man can travel the world by the air, the moon and the sun. ${ }^{17}$

What is called Zhenren, the Zhenren in the ancient time ... will not be trembling when climbing the highs, will not be wet when swimming in the water, and will not feel hot when going into fire. ${ }^{18}$

Such description of super power as "will not be wet when swimming in the water, and will not feel hot when going into fire" in fact shows a sense of life and ideals that people hope to get rid of restriction of expediency for life, so it forms a strong contrast with that the common people change "Zhi" and "oppose to or comply with things" due to expediency.

Generally, in the light of Zhuangzi's view, "Zhi" is originally the "Tain Yu" that people boast, but is used as tool and means by people for their expediency, rather than taking "Tian" as standard, so that "Zhi" is changed into a lethal weapon for fight. Just for this, Zhuangzi uses "Nie" to describe "Zhi", giving out his sign of "Zhi is evil". The above is the basic criticism and reflection of Zhuangzi for the "Zhi" among common people.

\footnotetext{
15 Guo Qingfan: "Zhuangzi Ji Shi ", p228-231

16 Guo Qingfan: "Zhuangzi Ji Shi ", p28

17 Guo Qingfan: "Zhuangzi Ji Shi ", p96

18 Guo Qingfan: "Zhuangzi Ji Shi ", p226
} 


\section{ZHENREN's “ZHI AT THE JUST TIME”}

People change "Zhi" as tool for their expediency, and reversely, Zhuanzi think Zhenren treats "Zhi"as "Zhi at the just time", and he made such description in the Great Master:

(As ancient Zhenren) a man regards the criminal law as the subject, then when he kills person, we think his generous and kind; a man regards the ritual as wings, he will act with the edification of etiquette in the world; a man waits for the opportunity with his knowledge, because of various things out of the way. Follow the rules with morality, as if all the persons with feet can climb the hills, but people think those persons are diligent in walking. So what people like is the same and what they dislike is the same; those same things are integrated and those different are also integrated. Those same things are similar to the nature, and those different things are similar with human. It is impossible for nature and human to be opposite but transcend each other. If understanding these, the man will be "Zhenren". ${ }^{19}$ In this regard, Guo Xiang said in his note: "criminal law is the rule to govern a county, not at one's pleasure; rite, is the convention forming naturally in the life, not formulated by people. The wise, acts at the opportunity, not advised by others; the virtuous, willingly follows the rite, not forced by others..." Corresponding to the first paragraph in "De Chong Fu"at the start of the first sub-section of this paper, Zhuangzi discussed "Xing", "Li"20, "Zhi", "De" from the positive aspect. From the note of Guo Xiang, we can see that Zhuangzi's essential and uniform idea is "not me" in his discussion of "Xing", "Li", "Zhi", "De", which form a vivid contrast to the artificial making of "me" due to our dependency. For Zhenren, "Xing", "Li", “Zhi”, "De”are all produce from the nature based on all things in the world (including human society), which is the truth in all things. Those people are called as Zhenren, because they follow the nature and take the nature as the organic part of their life, not artificial. Just for this, Zhenren can not dispute with the world but unite it so as to achieve such a life modality both as said by "following the law of the universe and grasping the change of "six qi" (refers to the yin, yang, wind, rain, darkness, brightness), traveling in the endless territory" ${ }^{, 1}$ in the Happy Tour and as said by "contacting with the world and spirit, but not disdain any thing in the world" in The World.

Concretely for this proposition of "Zhi at the just time", Zhuangzi made note to explain that "a man waits for the opportunity with his knowledge, because of various things out of the way", of which "various things out of the way" is used to explain "the opportunity" and "out of the way" is used to refer to the trend of the nature in the world. For the trend of the nature, Zhuangzi gives out the answer program is "compliance with it".

Zhuangzi's thought of compliance with natural trend, in fact means that a man should not produce the feeling of like or dislike due to his selfish mind when the natural trend is

\footnotetext{
19 Guo Qingfan: "Zhuangzi Ji Shi ", p234-235

20 Guo Qingfan: "Zhuangzi Ji Shi ", p238-240

21 As we think, the "Xing" and "Li" here may correspond to the "Yue" in the first paragraph of the "De Chong Fu" cited in the first section.
}

favorable or not conducive to him, but he should have a life feeling of "the monstrous flood can not drown him; the serious drought can make the stone melt and the earth burnt but he did not feel hot". So the idea of "compliance with the opportunity" and "compliance with the fortune" is actually to abolish the utilitarian and imposition of "Zhi", and also to cancel the utilitarian and imposition of human in life so as to pursue a life ideal of integration with the nature and all things in the world. By this, it can be seen that the core connotation of "Zhi at the just time" is to regard "Zhi" as that is compliance with the trend of nature and has no utilitarian and imposition for expediency. Obviously, for Zhuangzi, such "Zhi" without utilitarian and imposition must not be regarded as a tool and means that can be imposed by people, and it should be regarded as a purpose, that is to say, as the "Tian Yu" and "Tian Shi" endowed granted by the God, this "Zhi" is not for a special private purpose, but it constitutes a life basis of harmony between human and all things in the world, and the life ideal of "contacting with the world and spirit" can be realized only on the base of such a "Zhi". At this point, "Zhi" itself constitutes a way of existence for human, rather than a certain tool or means for human existence. Therefore, the "Zhi" in Zhenren's "Zhi at the just time" is also the "Zhi" praised by Zhuangzi in the words of "as big as can become a independent sky" in De Chong Fu, while the "Nie Zhi" described in the first section of this article is only the "Zhi" of "so small are people".

Just because the above-mentioned "Zhi" in Zhenren's "Zhi at the just time"itself constitutes the organic part of human existence and the life ideal, Zhuangzi said in the Great Master that "there is real Zhi after Zhenren",22 and we can get ride of the selfish feeling and mind with features of tools in our life only after having the life ideal of "Zhenren" and "contacting with the world and spirit", and further we can remove the utilitarian and imposition of "Zhi" to make the "Zhi" of "Tian Yu" become the "Zhi" of human existence and life ideal and the foundation of harmony between human and all things in the world.

At the same time, just because such Zhenren's "Zhi” removes the utilitarian and imposition of the "Zhi" among common people, human can comply with the trend of the nature in the world. Therefore, in the thought of Zhuangzi, it is considered a kind of "Zhi" in "no dependence". Zhuangzhi made definition of Zhenren's life realm in the Happy Tour:

As for the law of the universe to follow, if grasping the change of "six qi" (refers to the yin, yang, wind, rain, darkness, brightness), you can travel in the endless territory, and you need to rely on nothing! ${ }^{23}$

Before Zhuangzi elaborated the supreme realm of "no dependence" in the Happy Tour, the people from the person "having the ability competent for an official" to Liezi, all have their special goal for selfish life pursuit, so the life process including its "Zhi" ultimately becomes the tool to realize this specific goal for expediency, and only this goal is produced from their selfish pursuit. Form the viewpoint of

\footnotetext{
22 Guo Qingfan: "Zhuangzi Ji Shi ", p226

23 Guo Qingfan: "Zhuangzi Ji Shi ", p17
} 
Zhuangzi, it is out of selfishness, and thus must be limited, "finite" and behind the "spirit of heaven and earth ". In contrast, only when getting rid of utilitarian and imposition of the "Zhi", abolishing the solipsism, utilitarian and imposition in life, not absurdly producing "selfish" idea, we can make "Zhi" of mind conform to the trend of the nature and further make the spirit of human life not bound. Just because of the "necessary" trend of the nature but not of individual limited and temporary selfishness, Zhuangzi this "Zhi" can also be called "Zhi" of "no dependence", so as to distinguish it from the "Zhi" "with dependence" of common people for their selfishness.

On the basis of the insight for the above-mentioned "no dependence" of real "Zhi" and "with dependence" of evil "Zhi", Zhuangzi opposes the argument of "right and wrong" for "with dependence" but holds the idea of "not asking for right or wrong" 24 . From Zhuangzi's view, it may be the "Zhi" of "no dependence" for people to meet the trend of the nature, must not be the "Zhi" of "with dependence" due to selfishness. In this way, if people are able to have the "Zhi" in accordance with the trend of the nature, they will have a feelings of life in accordance with all things in the world, and then this "Zhi" is of "no dependence", thus people will not have "wrong" in the contrast, and there will not be the called argument of "right and wrong" among them; whereas if people have the "Zhi" due to their selfishness, the "Zhi" is right for their own expediency but wrong for others' expediency, then whether the "right" or the "wrong" is based on dependence, so the argument of "right" and "wrong" will produce and people always are cautious at the beginning of things. Zhuangzi does not recognize this evil "Zhi"due to selfishness and also not agree with the argument of "right and wrong". Zhuangzi expressed the above thought in the " Essay on the Uniformity of All Things ", and finally concluded that the way to solve the argument of "right and wrong" of the people is only to jump out of the argument of "right and wrong" itself and return to the "Tian $\mathrm{Ni}$ " of "no dependence". He said:

What is the natural way to reconcile with the world? The answer is: yes, it contains not; this contains not this. Yes, if it is so, then it is different from not, unnecessary for argument; so, if it is really so, then this is different from not this, unnecessary for argument. Make the same treatment with harmonious mind, but if can not be treated with the same way, use the natural rules to reconcile it, and follow the natural changes. So you can enjoy a life, forget the age, forget the right and wrong, excited in the infinite realm without struggle, then you will put itself in the leisure. ${ }^{25}$

Guo Xiang said in the note that we can finally achieve harmony with all things in the world and make our life in "the infinite realm" and make us "as big as to solely support a sky" only when people get rid of evil "Zhi" and return to the state of real "Zhi" to meet the trend of the nature. ${ }^{26}$ In a word, the concrete connotation of Zhenren for "Zhi at the just time" is to meet the trend of the nature and reconcile

24 Guo Qingfan: "Zhuangzi Ji Shi ", p1099

25 Guo Qingfan: "Zhuangzi Ji Shi ", p108

26 Guo Qingfan: "Zhuangzi Ji Shi ", p109 with all the things in the world, and this "Zhi" constitutes the life foundation of harmony between human and the nature for the life ideal. Compared with the "Zhi" of common people for selfishness, this "Zhi" abolishes all solipsism, utilitarianism, and imposition to completely integrate with all things in the world. The above can be regarded as the basic interpretation of Zhuangzi for the "Zhi" of Zhenren as "Tian Yu" and "Tian Shi ".

\section{CORRECTION OF PUBLIC "ZHI": "ZHI STOPS"}

Through the analysis of the "Zhi" of common people criticized by Zhuangzi and the "Zhi” of Zhenren recognized by him in the first two sections, we can clearly find that for Zhuangzi, the true "Zhi" is a intrinsic link in the process of the ideal of life that he practses his " integrating with all things in the world" and "contacting with the world and spirit", and it is an inseparable part of his life process, so he regards "Zhi" as "Tian Yu" and "Tian Shi". In this regard, it is not possible to make abstract discussion of "Zhi" out of the ideal of life, and not to vulgarly take "Zhi" as a tool and a means of personal selfishness. And the sadness of common people is for this, because they often regard the life as a kind of existence of selfishness and take the "Zhi" as a powerful means to benefit this selfishness. Therefore, they continuously expand this "Zhi" as tool and means to achieve their self-interest, but according to the logic, Zhuangzi made the elaboration in the Health Lord:

Our life is limited, and "Zhi" (knowledge) is infinite; when we pursue the infinite things with our limited life, there will be no good result. And when we achieve it, all die. ${ }^{27}$

Here, Zhuangzi demonstrated the absurdity according to the logic of common people: if pursuing the infinite "Zhi" of selfishness with the limited life, we will serve the things in a lifetime and has no return. We want the benefit but lose the most important thing in our life, so we say "all die".

The warning of "when we achieve it, all die" for the public is based on the theory of real knowledge. Zhuangzi remind us that the correct attitude towards "Zhi" should be "to realize the limit of knowledge".

The sage's sayings and argument of all things in the world are made "because of various things out of the way ", only for complying with the trend of the nature ("Tian Yu"), so Zhuangzi said "the sage thinks about things in mind" but the words and argument of common people are often to achieve the special goal of selfishness; because of a certain special goal to "fight", the words and argument of common people are to "debate for revealing this truth". Only when abandoning the "Zhi" of imposition that is the words and argument of "debating for revealing the truth", can common people promote their life realm and silently comply with the trend of the nature like the sage. If there is the "real Zhi", we will not have the offshoot and superabundant "evil Zhi" due to the selfishness, and such attitude to keep silent and not to debate for revealing the truth is "to realize the limit of knowledge" of Zhuangzi.

27 Guo Qingfan: "Zhuangzi Ji Shi ", p115 
Probably to warn the public not to fabricate the "Zhi" for the selfishness, "Zhi" appears mostly in negative sentences and questions, of which the most famous is the fable "continuous three-not-know to answer the questions" in the Essay on the Uniformity of All Things.

In this fable, Zhuangzi adopts Wang Ni's words to expatiate his view that he thinks the "Zhi of interest" as tool of selfishness can not be regarded as "Zhi". If there is not the ideal of life such as "such a man can travel the world by the air, the moon and the sun" and "contacting with the world and spirit ", we will simply not understand the meaning of "real Zhi", and even if spoken, it will be immediately misunderstood by the public. Just for this reason, Zhuangzi used Wang Ni's words "continuous three-not-know to answer the questions" to express his meanings to talk for the moment and then first give the hint of "what you do not know is real awareness but what you know is not always real awareness" to illuminate the difficulty to get ride of evil Zhi and obtain the real Zhi.

\section{CONCLUSION}

To sum up, according to the "Zhi of interest" of common people and the logic of the public pursuing the "Zhi" for their selfishness, Zhuangzi demonstrated the absurdity of their pursuit that "when we achieve it, all die", and on the other hand, he emphasized that for "real Zhi", people should comply with the trend of the nature and further gave the advice of "realizing the limit of knowledge" to tell people not artificial behaviors due to selfishness. We can say the above is the effort made by Zhuangzi to hope all the people get out of the "very little" pattern of life and achieve the realm of life "as big as can become an independent sky".

\section{REFERENCES}

[1] Lyu Huiqing [Song Dynasty]. "Zhuangzi Yi Ji Xiao" (Beijing: Zhonghua Book Company, 2009).

[2] Guo Qingfan [Qing Dynasty]. "Zhuangzi Ji Shi" (Beijing: Zhonghua Book Company, 2004).

[3] Lu Shuzhi [Qing Dynasty]. "Zhuangzi Xue" (Shanghai: East China Normal University Press, 2011).

[4] Zhong Tai. "Zhuangzi Fa Wei" (Shanghai: Shanghai Ancient Books Publishing House, 1988). 